\title{
Do Patients Return to Work After Total Knee Arthroplasty?
}

\author{
Adolph V. Lombardi Jr MD, Ryan M. Nunley MD, Keith R. Berend MD, \\ Erin L. Ruh MS, John C. Clohisy MD, William G. Hamilton MD, \\ Craig J. Della Valle MD, Javad Parvizi MD, Robert L. Barrack MD
}

Published online: 13 June 2013

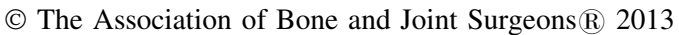

\begin{abstract}
Background Although there is extensive literature supporting a high success rate, there are limited data on return to work after total knee arthroplasty (TKA).

Questions/purposes We sought to determine (1) the percentage of patients who returned to work after TKA; (2) what percentage returned to their original job; (3) whether this varied based on the physical job demand category; and (4) whether there were differences between men and women in terms of the likelihood of returning to work after TKA.

Methods A multicenter study was conducted of patients of working age (18-60 years) who underwent TKA 1 to
\end{abstract}

The institution of one or more of the authors has received funding from Biomet Inc (Warsaw, IN, USA) (AVL, RMN, KRB, WGH, RLB), EOS Imaging Inc (Cambridge, MA, USA) (RMN, RLB), Medical Compression Systems, Inc (West Hills, CA, USA) (RMN, RLB), the National Institutes of Health (Bethesda, MD, USA) (JP, RLB), Smith \& Nephew, Inc (Memphis, TN, USA) (RMN, CDV, JP, RLB), Stryker Orthopaedics (Mahwah, NJ, USA) (AVL, RMN, CDV, JP, RLB), Wright Medical Technology, Inc (Arlington, TN, USA) (RMN, JCC, RLB), DePuy, a Johnson and Johnson Company (Warsaw, IN, USA) (WGH, JP), 3 M (St Paul, MN, USA) (JP), Baxter Healthcare Corp (Deerfield, IL, USA) (JP), Musculoskeletal Transplant Foundation (Edison, NJ, USA) (JP), Zimmer Inc (Warsaw, IN, USA) (JCC, JP), and Inova Health Care Services (Falls Church, VA, USA) (WGH). The institutions of the authors have received funding from Stryker Orthopaedics in support of this study. One of the authors certifies that he (AVL) has or may receive payments or benefits, during the study period, an amount of more than USD $1,000,001$ from Biomet Inc, and an amount of USD 10,000 to 100,000 from Innomed, Inc (Savannah, GA, USA). One of the authors certifies that he (RMN) has or may receive payments or benefits, during the study period, an amount of less than USD 10,000 from Smith \& Nephew, Inc, an amount of less than USD 10,000 from Wright Medical Technology, Inc, an amount of less than USD 10,000 from Medtronic (Minneapolis, MN, USA), an amount of less than USD 10,000 from CardioMEMS (Atlanta, GA, USA), and an amount of less than USD 10,000 from Integra LifeSciences (Plainsboro, NJ,
5 years previously. An independent third-party survey center with expertise in collecting healthcare data for state and federal agencies collected the data. Definitions from the US Department of Labor's Dictionary of Occupational Titles were used to determine physical job demand categories of sedentary, light, medium, heavy, and very heavy. Complete data were collected on 661 patients undergoing TKA (average age 54 years, $61 \%$ women).

Results Seventy-five percent (494 of 661) were employed in the 3 months before their TKA and 98\% (482 of 494) returned to work after surgery; $89 \%$ (440 of 494 ) returned to the same job. Before surgery, physical demand categories of

USA). One of the authors certifies that he (KRB) has or may receive payments or benefits, during the study period, an amount of more than USD 1,000,001 from Biomet Inc. One of the authors certifies that he (JCC) has or may receive payments or benefits, during the study period, an amount of less than USD 10,000 from Biomet Inc, and an amount of less than USD 10,000 from Pivot Medical (Sunnyvale, CA, USA). One of the authors certifies that he (WGH) has or may receive payments or benefits, during the study period, an amount of USD 100,001 to 1,000,000 from DePuy, a Johnson and Johnson Company, and an amount of USD 10,000 to 100,000 from Medtronic. One of the authors certifies that he (CDV) has or may receive payments or benefits, during the study period, an amount of USD 10,000 to 100,000 from Biomet Inc, an amount of USD 10,000 to 100,000 from Smith \& Nephew, Inc, an amount of less than USD 10,000 from ConvaTec (Skillman, NJ, USA), and an amount of USD 10,000 to 100,000 from CD Diagnostics (Wynnewood, PA, USA). One of the authors certifies that he (JP) has or may receive payments or benefits, during the study period, an amount of USD 10,000 to 100,000 from Smith \& Nephew, Inc, an amount of USD 10,000 to 100,000 from $3 \mathrm{M}$, an amount of USD 10,000 to 100,000 from Cadence Pharmaceuticals, Inc (San Diego, CA, USA), an amount of USD 10,000 to 100,000 from CeramTec (Laurens, SC, USA), an amount of USD 10,000 to 100,000 from Pfizer (New York, NY, USA), an amount of USD 10,000 to 100,000 from Salient Surgical (Minneapolis, MN, USA), an amount of USD 10,000 to 100,000 from TissueGene (Rockville, MD, USA), and an amount of USD 10,000 to 100,000 from Zimmer Inc. One of the 
the patients' jobs were sedentary $12 \%$, light $10 \%$, medium $24 \%$, heavy $24 \%$, and very heavy $30 \%$. The return to work rate for those employed during the 3 months before surgery by physical demand category was sedentary $95 \%$, light $91 \%$, medium $100 \%$, heavy $98 \%$, and very heavy $97 \%$. Men were more likely than women to have worked within the 3 months before TKA but there was no difference between sexes in return to work after TKA.

Conclusions In this group of young patients, most returned to work at their usual occupation after TKA.

Level of Evidence Level IV, therapeutic study. See Guidelines for Authors for a complete description of levels of evidence.

\section{Introduction}

TKA is among the most widely performed and widely studied of medical procedures. It has been highly successful in relieving pain and restoring function in patients with advanced arthritis of the knee [12]. It is well known that many patients who have undergone joint arthroplasty benefit from improved pain relief, mobility, and functional demands of daily living. It is expected over the next 10 years that there will be a significant increase in the number of TKAs performed in the United States and throughout the world [5, 7]. Although in the past TKA has been reserved for older and more sedentary patients, the demand for return to active lifestyles has pushed many patients and physicians to perform these procedures on patients who are young and working $[11,16]$. Demand for primary TKA in patients

authors certifies that he (RLB) has or may receive payments or benefits, during the study period, an amount of more than USD 1,000,001 from Smith \& Nephew, Inc, and an amount of more than USD 1,000,001 from Stryker Orthopaedics.

Each author certifies that his or her institution approved the human protocol for this investigation, that all investigations were conducted in conformity with ethical principles of research, and that informed consent for participation in the study was obtained.

All ICMJE Conflict of Interest Forms for authors and Clinical Orthopaedics and Related Research editors and board members are on file with the publication and can be viewed on request.

This work was performed at Washington University School of Medicine (St Louis, MO, USA), Joint Implant Surgeons, Inc (New Albany, OH, USA), Rothman Institute of Orthopedics at Thomas Jefferson University (Philadelphia, PA, USA), Midwest Orthopaedics at Rush (Chicago, IL, USA), and Anderson Orthopaedic Clinic (Arlington, VA, USA).

Clinical Orthopaedics and Related Research neither advocates nor endorses the use of any treatment, drug, or device. Readers are encouraged to always seek additional information, including FDAapproval status, of any drug or device prior to clinical use.

Each author certifies that his or her institution has approved the human protocol for this investigation, that all investigations were conducted in conformity with ethical principles of research, and that informed consent for participation in the study was obtained. younger than 65 years old is projected to exceed $50 \%$ of all patients undergoing TKA by 2016 [6], and many of these patients will be in the workforce at the time they have the procedure. There are limited data on return to work after TKA [2-4, 9, 10, 13, 17-19].

A similar multicenter, retrospective study involving four of the five centers in the current study was performed to evaluate the effect of hip arthroplasty (including total and resurfacing hip arthroplasty) on patients' ability to work and return to work [14]. The authors found that $90 \%$ of young, active patients returned to work after hip arthroplasty with the vast majority returning to their preoperative occupation and very few limited in their ability to return to work because of their operated hip.

In this study, we sought to determine (1) the percentage of patients who returned to work after TKA; (2) what percentage returned to their original job; (3) whether this varied based on the physical job demand category; and (4) whether there were differences between men and women in terms of the likelihood of returning to work after TKA.

\section{Patients and Methods}

Before the start of this study, institutional review board consent was obtained at Washington University to serve as the coordinating center. Four other institutions and an independent third-party survey center (University of Wisconsin Survey Center, Madison, WI, USA) were enlisted to participate. This telephone research survey company specializes in administering telephone questionnaires

A. V. Lombardi Jr ( $₫)$, K. R. Berend

Joint Implant Surgeons, Inc, 7277 Smith's Mill Road, Suite 200,

New Albany, OH 43054, USA

e-mail: lombardiav@joint-surgeons.com

A. V. Lombardi Jr, K. R. Berend

The Ohio State University, Columbus, OH, USA

A. V. Lombardi Jr, K. R. Berend

Mount Carmel Health System, New Albany, OH, USA

R. M. Nunley, E. L. Ruh, J. C. Clohisy, R. L. Barrack

Washington University in St Louis School of Medicine, St Louis, MO, USA

W. G. Hamilton

Anderson Orthopaedic Clinic, Alexandria, VA, USA

C. J. Della Valle

Rush University Medical Center, Chicago, IL, USA

J. Parvizi

Rothman Institute at Thomas Jefferson University, Philadelphia, PA, USA 
for medical and scientific research. The additional institutions and survey center further obtained their own institutional review board approval. Each participating institution had a high volume of TKAs performed by an experienced core group of surgeons and each had a well-established joint registry in place; each center provided a geographically diverse mix of academic, referral, and community-based practice. All implants were approved for the uses described in this study by the US Food and Drug Administration. At each center, the determination for patients to return to work after TKA was based on each patient's course of recovery postoperatively and job physical demands. In general, patients employed in sedentary jobs were allowed to return to work whenever they could safely get there, generally at 2 to 6 weeks, and advised to elevate the operated extremity when possible and get up and walk around at appropriate intervals. Driving was allowed after discontinuation of narcotic pain medications, for the left extremity at the patient's discretion, and for the right extremity when capable of a safe brake response. For lightduty jobs that require more standing and walking, patients were allowed to return when they could walk without a limp, generally at 6 to 12 weeks. For heavy demand jobs, patients were allowed to return to work generally at 10 to 12 weeks, when they were capable of safely performing their job requirements. Each center makes exceptions for motivated patients who wish to return to work earlier than the general guidelines indicate.

Each investigation center evaluated their knee surgery registry and compiled a consecutive list of patients who fulfilled the inclusion and exclusion criteria for the study. The multicenter study was conducted of male and female patients of working age who underwent TKA 1 to 3 years previously, from July 2007 through July 2010, and had a minimum of 1-year followup. Inclusion criteria were (1) men or women at least 18 years of age and no older than 60 years of age at surgery, inclusive, and skeletally mature; (2) patients requiring primary knee surgery as a result of noninflammatory arthritis (degenerative joint disease) such as osteoarthritis, traumatic arthritis, or avascular necrosis; (3) patient's index surgery was 1 to 3 years before the start of the study; (4) the patient was willing to consent to participate in the study; and (5) the patient met none of the exclusion criteria. Exclusion criteria were (1) patients with a history of previous infection or sepsis in the knee or fracture, dislocation, or revision to the operated knee; and (2) patients with extensive medical comorbidities including hypertension in combination with other comorbidities, renal failure, coronary artery disease, liver disease, sickle cell disease, inflammatory arthropathy, respiratory disease, cancer, etc, that would limit their activity level.

Each center then mailed its eligible patients a standard letter describing the research study. The letter explained the goals of the study and that the patient would be receiving a telephone call from a specially trained interviewer located at the University of Wisconsin. The identified patients from all five sites were reviewed and compiled by the coordinating center and forwarded to the independent third-party telephone research survey company, which then administered an unbiased and blinded telephone knee function assessment questionnaire. This independent research survey company was not affiliated with any of the five investigational sites and has expertise in collecting healthcare data for state and federal agencies. Patients were not offered any incentive to participate in the study.

The survey questionnaire was designed by the coordinating center with assistance from the third-party telephone survey center with the purpose of assessing objective outcomes important to high-demand patients after undergoing TKA and is essentially the same as the questionnaire used in the aforementioned earlier study of patients undergoing hip arthroplasty [14]. The questionnaire used a unique skip logic preprogrammed algorithm (Appendix 1) to compare pre- and postoperative outcomes related to employment. Definitions from the US Department of Labor's Dictionary of Occupational Titles (DOT) were used to determine physical job demand categories of sedentary, light, medium, heavy, and very heavy (Table 1) [1]. Although the DOT was replaced in 1998 by an online database, Occupational Information Network $(O * N E T)$ [15], data from the 1991 revised fourth-edition DOT are used extensively by the Social Security Administration for determining disability and are available online through the Office of Administrative Law Judges Law Library [1].

Interviews were conducted from August 2011 to April 2012, and all interviews were conducted in English. Interviewers called each patient at the telephone number(s) provided by their surgeon's institution. If successfully contacted, the interviewer asked the patients a series of screening questions to confirm eligibility for the study. The full questionnaire was administered to only those patients who gave verbal consent to participate, were confirmed to be eligible, and determined to be capable of participation based on their responses to the screening questions. The survey protocol called for 25 telephone call attempts per patient. Cases involving a refusal to participate were called back in an attempt to convert the refusal to a completed interview. However, no further attempts were made if a second refusal occurred, and conversion was not attempted after irate refusals. Interviews were performed using a computer-based telephone interviewing system featuring CASES 4.3.7 software (Computer-Assisted Survey Methods Program at the University of California-Berkeley, Berkeley, CA, USA). With the CASES software, the text of the survey appears question by question on a computer screen for the interviewer to read to the respondent. 
Table 1. Physical job demand categories based on definitions from the United States Department of Labor's Dictionary of Occupational Titles (DOT) [1]

\begin{tabular}{|c|c|c|}
\hline Work category & Definition & Examples \\
\hline Sedentary & $\begin{array}{l}\text { Exerting up to } 10 \text { pounds of force occasionally (occasionally: activity or } \\
\text { condition exists up to one-third of the time) and/or a negligible amount of } \\
\text { force frequently (frequently: activity or condition exists from one-third to } \\
\text { two-thirds of the time) to lift, carry, push, pull, or otherwise move objects, } \\
\text { including the human body. Sedentary work involves sitting most of the time, } \\
\text { but may involve walking or standing for brief periods of time. Jobs are } \\
\text { sedentary if walking and standing are required only occasionally and all } \\
\text { other sedentary criteria are met. }\end{array}$ & $\begin{array}{l}\text { Receptionist, secretary, } \\
\text { accountant, tax preparer, } \\
\text { counselor, telemarketer, bill } \\
\text { collector, computer programmer }\end{array}$ \\
\hline Light labor & $\begin{array}{l}\text { Exerting up to } 20 \text { pounds of force occasionally, and/or up to } 10 \text { pounds of force } \\
\text { frequently, and/or a negligible amount of force constantly (constantly: } \\
\text { activity or condition exists two-thirds or more of the time) to move objects. } \\
\text { Physical demand requirements are in excess of those for sedentary work. } \\
\text { Although the weight lifted may be only a negligible amount, a job should be } \\
\text { rated light work: (1) when it requires walking or standing to a significant } \\
\text { degree; or (2) when it requires sitting most of the time but entails pushing } \\
\text { and/or pulling of arm or leg controls; and/or (3) when the job requires } \\
\text { working at a production rate pace entailing the constant pushing and/or } \\
\text { pulling of materials although the weight of those materials is negligible. } \\
\text { NOTE: The constant stress and strain of maintaining a production rate pace, } \\
\text { especially in an industrial setting, can be and is physically demanding of a } \\
\text { worker even though the amount of force exerted is negligible. }\end{array}$ & $\begin{array}{l}\text { Teacher, assembly line worker, } \\
\text { sewing machine operator, retail } \\
\text { clerk }\end{array}$ \\
\hline Medium labor & $\begin{array}{l}\text { Exerting } 20 \text { to } 50 \text { pounds of force occasionally, and/or } 10 \text { to } 25 \text { pounds of force } \\
\text { frequently, and/or greater than negligible up to } 10 \text { pounds of force constantly } \\
\text { to move objects. Physical demand requirements are in excess of those for } \\
\text { light work. }\end{array}$ & $\begin{array}{l}\text { Postal worker, maintenance } \\
\text { worker, taxi driver, bus driver }\end{array}$ \\
\hline Heavy labor & $\begin{array}{l}\text { Exerting } 50 \text { to } 100 \text { pounds of force occasionally, and/or } 25 \text { to } 50 \text { pounds of } \\
\text { force frequently, and/or } 10 \text { to } 20 \text { pounds of force constantly to move objects. } \\
\text { Physical demand requirements are in excess of those for medium work. }\end{array}$ & $\begin{array}{l}\text { Heavy machine operator, truck } \\
\text { driver, mechanic }\end{array}$ \\
\hline Very heavy labor & $\begin{array}{l}\text { Exerting in excess of } 100 \text { pounds of force occasionally, and/or in excess of } 50 \\
\text { pounds of force frequently, and/or in excess of } 20 \text { pounds of force constantly } \\
\text { to move objects. Physical demand requirements are in excess of those for } \\
\text { heavy work. }\end{array}$ & $\begin{array}{l}\text { Construction worker, farmer, } \\
\text { produce picker, stock clerk }\end{array}$ \\
\hline
\end{tabular}

The interview is routed based on preprogrammed skip logic that allows for precoded questions, open-ended questions, and combinations of the two.

The initial cohort consisted of 1139 consecutive patients who underwent TKA and met the inclusion criteria to participate in the survey. Of those, there were 112 screen failures, 54 refusals at the household level, and 69 participant refusals. An additional 114 participants were never available, 109 were not found as a result of a bad address/ phone number, eight had died, seven were contacted but did not complete the interview, and five had a language barrier that prevented them from completing the survey. This left 661 completed interviews for final analysis (response rate $64.4 \%$ ). The average patient age at the time of knee operation was 54 years (range, 19-60 years). Sex was female in 405 patients $(61 \%)$ and male in 256 (39\%). The operative side was the right in 336 knees $(51 \%)$ and left in 325 (49\%). Preoperative diagnosis was osteoarthritis in 646 knees (98\%), posttraumatic arthritis in $13(2 \%)$, and two avascular necrosis. Implant type was cruciate- retaining in 346 (52\%), posterior-stabilized in 181 (27\%), rotating platform in $61(9 \%)$, high flexion in $42(6 \%)$, and sex-specific in $31(5 \%)$. The average time to the interview after knee surgery was 2.6 years (range, $1-5$ years).

Of the 661 patients interviewed about their employment history, $494(75 \%)$ reported working for pay in the 3 months before their TKA, whereas 166 (25\%) reported they had not been working. Based on questions related to the physical demands of the last job they worked before undergoing TKA, the patients' job DOT physical demand categories before surgery were sedentary for 73 (12\%), light for $65(10 \%)$, medium for 151 (24\%), heavy for $149(24 \%)$, and very heavy for 187 (30\%) (Table 2). Of the 166 patients not working before surgery, the primary reason given was categorized as retired for $54(33 \%)$, unable to work 47 (28\%), keeping house 37 (22\%), looking for work three (2\%), and something else for 23 (14\%), whereas two patients did not state a reason. For the patients who were not working before their TKA, the time since they had last worked averaged 6.4 years (SD, 8.2; range, $0.01-45$ years). 
Table 2. Return to work by physical demand category of last job worked before TKA

\begin{tabular}{lllllll}
\hline $\begin{array}{l}\text { Physical } \\
\text { demand } \\
\text { category }\end{array}$ & $\begin{array}{l}\text { At last job worked } \\
\text { before TKA }\end{array}$ & $\begin{array}{l}\text { Working within } \\
\text { T months before } \\
\text { TKA }\end{array}$ & $\begin{array}{l}\text { Worked at all } \\
\text { after TKA }\end{array}$ & $\begin{array}{l}\text { Returned to usual } \\
\text { job after TKA }\end{array}$ & $\begin{array}{l}\text { Still working at } \\
1 \text { year after TKA }\end{array}$ & $\begin{array}{l}\text { Still working at } \\
\text { time of interview }\end{array}$ \\
\hline Very heavy & $187(30 \%)$ & $142 / 187(76 \%)$ & $138 / 142(97 \%)$ & $121 / 138(88 \%)$ & $135 / 138(98 \%)$ & $125 / 138(91 \%)$ \\
Heavy & $149(24 \%)$ & $115 / 149(77 \%)$ & $113 / 115(98 \%)$ & $104 / 113(92 \%)$ & $109 / 113(96 \%)$ & $93 / 113(82 \%)$ \\
Medium & $151(24 \%)$ & $118 / 151(78 \%)$ & $121 / 118(102 \%)$ & $112 / 121(93 \%)$ & $115 / 121(95 \%)$ & $102 / 121(84 \%)$ \\
Light & $65(10 \%)$ & $53 / 65(82 \%)$ & $48 / 53(91 \%)$ & $43 / 48(90 \%)$ & $43 / 48(90 \%)$ & $37 / 48(77 \%)$ \\
Sedentary & $73(12 \%)$ & $65 / 73(89 \%)$ & $62 / 65(95 \%)$ & $60 / 62(97 \%)$ & $59 / 62(95 \%)$ & $52 / 62(84 \%)$ \\
Total & 625 & $493 / 625(79 \%)$ & $482 / 493(98 \%)$ & $440 / 482(91 \%)$ & $461 / 482(96 \%)$ & $409 / 482(85 \%)$ \\
p value & & 0.1933 & 0.0000 & 0.2767 & 0.1897 & 0.1696 \\
\hline
\end{tabular}

Statistical differences in return to work rates between sexes and physical demand categories were measured using chi-square analyses. A one-way analysis of variance was used to compare differences in mean years since last worked preoperatively, months worked with restrictions, weeks unable to work, and hours worked per week between the physical demand category groups, whereas Student's t-test was used to compare differences in these means between sexes. All $\mathrm{p}$ values were two-sided with $\mathrm{p}$ values $<0.05$ considered statistically significant. Ninety-five percent confidence intervals were used in all analyses.

\section{Results}

A large majority (98\%) of the 494 patients who were working during the 3 months before their TKA returned to work at some point after recovery from surgery. The 143 patients (23\%) who did not work at all after their TKA cite the following primary reasons: $66(46 \%)$ retired, $30(21 \%)$ were keeping house, 21 (15\%) were unable to work, 11 (8\%) were looking for work, one was a volunteer, one decided not to return to work for reasons not related to the operative knee, and $14(12 \%)$ for something else. Of the 21 patients who were unable to work, all were permanently disabled.

Of the 482 patients who worked at some point after undergoing TKA, 440 (91\%) were able to return successfully to their usual job either with or without restrictions, whereas $42(9 \%)$ patients did not return to the same job. For these patients who returned to work, the length of time for recovery after surgery averaged 8.9 weeks (SD, 9.1; range, 0-104 weeks). Ninety-two patients (19\%) reported having some type of temporary postoperative restrictions at work after surgery because of their knee operation, which lasted for an average of 3 months (SD, 4.0; range, 0.2-24 months).

When stratified by physical demand category, the rate of patients who were able to return to their usual job was not significantly different between groups (Table 2). For the 42 patients overall who did not return to their usual job, $11(26 \%)$ said the reason was primarily because of their operated knee. Patients employed at very heavy labor required the longest recovery period at 10.7 weeks compared with 8.1 weeks for both heavy and medium laborers, 10.5 weeks for light laborers, and only 6.5 weeks for sedentary laborers $(\mathrm{p}=0.011)$. When stratified by preoperative physical demand category, there was no difference between groups in either need for restrictions or length of time worked with postoperative restrictions.

For patients who worked in the 3 months before TKA, there was no difference between men and women in the percentage of patients who returned to work at any time after TKA (Table 3; $96 \%$ of men versus $99 \%$ of women, $\mathrm{p}=0.09$ ). An equal large majority (both $91 \%$ ) of men and women were able to return to the same job after TKA. Preoperative physical demand categories for men were $45 \%$ very heavy, $30 \%$ heavy, $18 \%$ medium, $7 \%$ light, and $10 \%$ sedentary, and for women were $20 \%$ very heavy, $26 \%$ heavy, $28 \%$ medium, $13 \%$ light, and $13 \%$ sedentary. Although men were the majority in the very heavy demand category and the largest category for women was medium demand (28\%), there were more women in the very heavy and heavy demand categories (46\%) than light and sedentary (26\%). There was no difference between sexes in the need for restrictions or length of time worked with restrictions either before or after TKA surgery or in time off work for recovery after surgery.

\section{Discussion}

The ability to return to work and continue working is an important goal for many patients undergoing TKA, especially for younger, more active, high-demand patients [11]. Employment is important not only for economic wellbeing, but for many individuals for mental well-being with work providing a sense of personal fulfillment, social 
Table 3. Return to work by sex

\begin{tabular}{lllllll}
\hline Sex & $\begin{array}{l}\text { Overall of patients } \\
\text { surveyed }\end{array}$ & $\begin{array}{l}\text { Working within } \\
\text { 3 months before TKA }\end{array}$ & $\begin{array}{l}\text { Worked at all } \\
\text { after TKA }\end{array}$ & $\begin{array}{l}\text { Returned to usual } \\
\text { job after TKA }\end{array}$ & $\begin{array}{l}\text { Still working at } \\
\text { 1 year after TKA }\end{array}$ & $\begin{array}{l}\text { Still working at } \\
\text { time of interview }\end{array}$ \\
\hline Females & $405(61 \%)$ & $282 / 405(70 \%)$ & $278 / 282(99 \%)$ & $254 / 278(91 \%)$ & $263 / 278(95 \%)$ & $231 / 278(83 \%)$ \\
Males & $256(39 \%)$ & $212 / 256(83 \%)$ & $204 / 212(96 \%)$ & $186 / 204(91 \%)$ & $198 / 204(97 \%)$ & $178 / 204(87 \%)$ \\
Total & 661 & $494 / 661(75 \%)$ & $482 / 494(98 \%)$ & $440 / 482(91 \%)$ & $461 / 482(96 \%)$ & $409 / 482(85 \%)$ \\
p value & & 0.0001 & 0.0924 & 1 & 0.1921 & 0.2047 \\
\hline
\end{tabular}

interaction, and achievement. Access to affordable health insurance for many patients is a benefit of employment in the United States and many other countries. In one US study of patients between the ages of 18 and 69 years undergoing TKA, 77\% were receiving health insurance through their employer [17]. Although TKA is one of the most commonly and increasingly performed surgical procedures in the world, there is limited information for younger, high-demand patients in regard to return to work (Table 4). The existing published studies on return to work after TKA are limited by several factors: (1) there is a wide range in expected recovery time after knee arthroplasty in published studies ranging from 4 to 9 weeks in three US studies [9, 17, 18] to as high as 12 months in one study [2]; (2) the patient cohorts were small $(<120$ TKAs) $[2,4,9,10]$; (3) the average patient age was older than 65 years [3, 13, 18, 19]; (4) the study included low-demand patients [3, 4, $13,18,19]$; (5) the study was reported in the literature more than 10 years ago [4, 13, 19]; and (6) the study was not designed specifically to analyze return to work [3, 9, 19]. The purpose of this multicenter study was to determine whether young patients who were working before undergoing primary TKA are able to return to work postoperatively and to determine whether the patient's preoperative job physical demand category has an effect on ability to return to work.

This study has several limitations. This was a retrospective study, performed by interviewing by telephone patients who were more than 1 year postoperative from TKA, which raises the potential for recall bias [8]. To limit this concern, we set a maximum target for recruitment into the study of patients no more than 3 years after their primary surgery, which in some cases approached 5 years by the time the study was completed. Another limitation of the study is the lack of a $100 \%$ response rate. Despite multiple attempts to contact all patients, $36 \%$ of the patients did not complete the survey. This was a multicenter study with TKA performed by several surgeons in a geographically diverse group of locations. Therefore, we could not control for differences in patient selection, implant selection, regional differences in local industries and job market, and specific advice given to patients regarding work limitations and return to work after surgery. However, all surgeons were experienced, highvolume arthroplasty specialists. By including a large number of patients from a diverse group of geographic locations within the United States, we sought to give a representational cross-section of the population. We adhered to a strict set of inclusion and exclusion criteria, excluding patients with inflammatory arthropathies and significant comorbidities that might limit their postoperative activity level and confound observations regarding the effects of TKA. The downturn in the US economy and higher unemployment rates may have impacted the ability of study patients to find work before and after surgery. Our surgeries were performed between 2008 and 2010, which coincides with the US economic downturn. Of patients who did not work after their knee surgery, $8 \%$ said they had looked for work.

In our study of patients younger than 60 years old undergoing TKA, a large majority of patients working before surgery were able to return to work after surgery, and most were able to return to their usual job after TKA, even very heavy demand jobs.. Patients working in very heavy jobs required the most time off for recovery, an average of 11 weeks, whereas those employed in sedentary positions averaged only 7 weeks for recovery. These recovery periods are consistent with the general recommendations of the participating surgery centers. Our overall average of time of 9 weeks off work for recovery after TKA is the same as that reported in another American study involving 162 patients undergoing TKA, all of whom were working before surgery and followed prospectively for 6 months after surgery [17]. The percentage of patients in our study who required restrictions after surgery (19\%) was similar to those requiring restrictions before TKA (18\%). The highest percentage of patients by physical job demand category in our study who were still working at 1 year after TKA and when surveyed were those in very heavy demand jobs.

In our study there was no difference between male and female patients in either the percentage who returned to work or in time off work required for recovery after surgery. When assessed by physical demand category, recovery time between men and women was the same in the very heavy, medium, and light categories, but faster for men than women in the heavy and sedentary groups. This is contrast to the findings of Styron et al. [17] in their recent prospective US-based study of 162 young, working patients undergoing TKA. Although their overall recovery time was the same as in our study, women returned to work much 


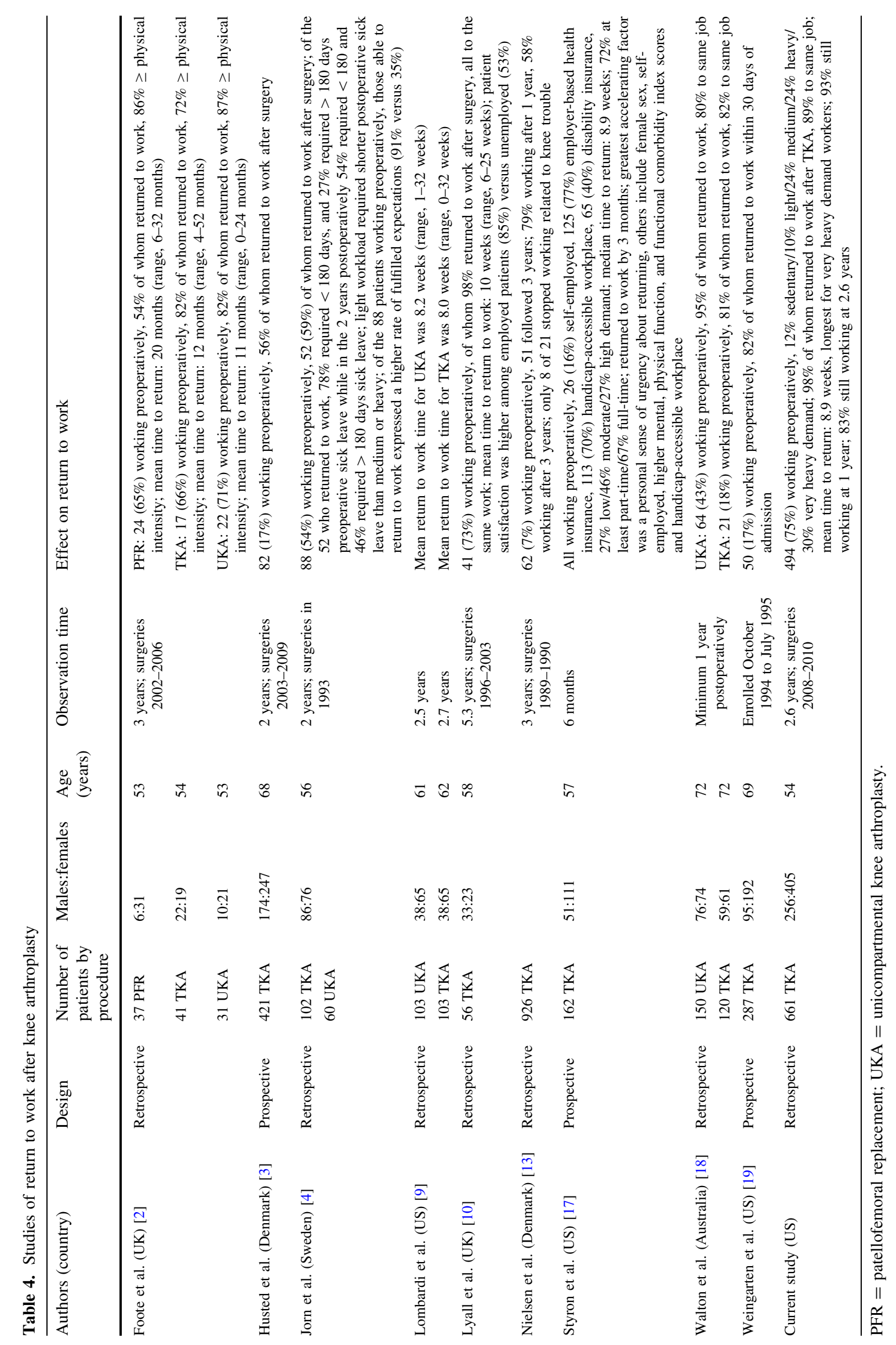


faster than men independent of physical demand category or obesity.

The ability to return to work is an important expectation for many patients considering TKA [11]. In this group of young, active patients undergoing TKA, most returned to work at their usual occupation. Although those patients with sedentary occupations had the highest rate of return to their usual work (97\%), even those with very heavy jobs returned to their same job $88 \%$ of the time. For purposes of planning for expenses, leave time, and job coverage, time off work for recovery from surgery averaged 2 months overall and 2.5 months for workers in very heavy demand jobs.

Appendix 1. Telephone questionnaire: section related to work

1. In the 3 months before your knee operation, did you work for pay at all?

a. If no, were you looking for work, keeping house, unable to work, retired, volunteering, or something else?

b. If no, were you temporarily unable to work or are you permanently disabled?

c. If no, what were you doing?

d. If no, when you had your knee operation in [month, year], how long had it been since you last had worked?

2. In the month before your knee operation, about how many hours per week did you work?

3. In the month before your knee operation, did you do your usual work or different work?

4. In the month before your knee operation, did you have any restrictions for your work because of your knee?

5. Before your knee operation, for about how long did you have any restrictions for your work because of your knee?

6. In the last job you had before your knee operation, what kind of work did you do?

7. What were your principle activities or duties?

8. What kind of business or industry was this?

9. Was this mainly manufacturing, wholesale trade, retail trade, or something else?

10. Were you employed by government, by a private company or organization (including nonprofit firms), or were you self-employed or working in a family business?

11. Was this business incorporated?

12. The next questions are about how demanding the last job you had before your operation was physically. In the last job you had before your knee was operated on: a. How often did you lift items weighing more than 100 pounds?

b. How often did you lift items weighing between 50 and 100 pounds?

c. How often did you lift items weighing between 20 and 50 pounds?

d. How often did you lift items weighing between 10 and 20 pounds?

e. How often did you lift items weighing up to 10 pounds?

13. In the last job you had before your knee was operated on, did you stand or walk one-third of the time or less or more than one-third of the time?

14. In the last job you had before your knee was operated on, would you describe the physical demands of the job as very light, light, medium, heavy, or very heavy?

15. Since your knee operation, have you worked at all?

a. If no, have you looked for work, kept house, been unable to work, been retired, been volunteering, decided not to return to work, or something else?

b. If no, are you or were you temporarily unable to work or are you permanently disabled?

c. If no, did you decide not to return to work primarily because of your operated knee?

d. If no, what did you do?

16. After your knee operation, for how many weeks were you unable to work as a result of your operation?

17. After your knee operation, did you ever return to the usual job you had before your knee operation either with or without restrictions?

a. If no, after your knee operation did you not return to your usual job primarily because of your operated knee?

18. After your knee operation, did you ever have any restrictions for your work because of your knee operation?

a. If yes, after your knee operation, for about how long did you have any restrictions for your work because of your operated knee?

19. At this time are you still working?

a. If no, are you still not working primarily because of your operated knee?

b. If no, were you still working 1 year after your knee operation?

20. In the last 30 days, how many hours per week did you work?

21. One year after your knee operation, were you working in the usual job you had before your knee operation? 
22. In the job you had 1 year after your knee operation, what kind of work did you do?

23. What were your principle activities or duties?

24. What kind of business or industry was this?

25. Was this mainly manufacturing, wholesale trade, retail trade, or something else?

26. Were you employed by government, by a private company or organization (including nonprofit firms), or were you self-employed or working in a family business?

27. Was this business incorporated?

28. The next questions are about how demanding the job you had 1 year after your operation was physically. In the job you had 1 year after your knee was operated on:

a. How often did you lift items weighing more than 100 pounds?

b. How often did you lift items weighing between 50 and 100 pounds?

c. How often did you lift items weighing between 20 and 50 pounds?

d. How often did you lift items weighing between 10 and 20 pounds?

e. How often did you lift items weighing up to 10 pounds?

29. In the job you had 1 year after your knee was operated on, did you stand or walk one-third of the time or less or more than one-third of the time?

30. In the job you had 1 year after your knee was operated on, would you describe the physical demands of the job as very light, light, medium, heavy, or very heavy?

\section{References}

1. Dictionary of Occupational Titles (DOT). Revised $4^{\text {th }}$ ed. US Department of Labor, Office of Administrative Law Judges; 1991. Available at: www.oalj.dol.gov/PUBLIC/DOT/REFERENCES/ DOTAPPC.HTM. Accessed December 4, 2012.

2. Foote JA, Smith HK, Jonas SC, Greenwood R, Weale AE. Return to work following knee arthroplasty. Knee. 2010;17:19-22.

3. Husted H, Troelsen A, Otte KS, Kristensen BB, Holm G, Kehlet H. Fast-track surgery for bilateral total knee replacement. $J$ Bone Joint Surg Br. 2011;93:351-356.

4. Jorn LP, Johnsson R, Toksvig-Larsen S. Patient satisfaction, function and return to work after knee arthroplasty. Acta Orthop Scand. 1999;70:343-347.

5. Kurtz S, Ong K, Lau E, Mowat F, Halpern M. Projections of primary and revision hip and knee arthroplasty in the United
States from 2005 to 2030. J Bone Joint Surg Am. 2007;89:780785.

6. Kurtz SM, Lau E, Ong K, Zhao K, Kelly M, Bozic KJ. Future young patient demand for primary and revision joint replacement: national projections from 2010 to 2030. Clin Orthop Relat Res. 2009;467:2606-2612.

7. Kurtz SM, Ong KL, Lau E, Widmer M, Maravic M, GómezBarrena E, de Pina Mde F, Manno V, Torre M, Walter WL, de Steiger R, Geesink RG, Peltola M, Röder C. International survey of primary and revision total knee replacement. Int Orthop. 2011;35:1783-1789.

8. Lingard EA, Wright EA, Sledge CB; Kinemax Outcomes Group. Pitfalls of using patient recall to derive preoperative status in outcome studies of total knee arthroplasty. J Bone Joint Surg Am. 2001;83:1149-1156.

9. Lombardi AV Jr, Berend KR, Walter CA, Aziz-Jacobo J, Cheney NA. Is recovery faster for mobile-bearing unicompartmental than total knee arthroplasty? Clin Orthop Relat Res. 2009;467:14501457.

10. Lyall H, Ireland J, El-Zebdeh MY. The effect of total knee replacement on employment in patients under 60 years of age. Ann R Coll Surg Engl. 2009;91:410-413.

11. Mancuso CA, Ranawat CS, Esdaile JM, Johanson NA, Charlson ME. Indications for total hip and total knee arthroplasties. Results of orthopaedic surveys. J Arthroplasty. 1996;11:3446.

12. Meding JB, Meding LK, Ritter MA, Keating EM. Pain relief and functional improvement remain 20 years after knee arthroplasty. Clin Orthop Relat Res. 2012;470:144-149.

13. Nielsen MB, Kristensen PW, Lamm M, Schrøder HM. [Knee alloplasty and working ability. The significance of knee alloplasty for working ability of patients who were working prior to surgery] [in Danish]. Ugeskr Laeger. 1999;161:26662669.

14. Nunley RM, Ruh EL, Zhang Q, Della Valle CJ, Engh CA Jr, Berend ME, Parvizi J, Clohisy JC, Barrack RL. Do patients return to work after hip arthroplasty surgery. J Arthroplasty. 2011;26(Suppl):92-98.e1-3.

15. Occupational Information Network $\left(\mathrm{O}^{*} \mathrm{NET}\right)$, US Department of Labor/Employment and Training Administration. Available at: www.onetonline.org/. Accessed December 4, 2012.

16. Ravi B, Croxford R, Reichmann WM, Losina E, Katz JN, Hawker GA. The changing demographics of total joint arthroplasty recipients in the United States and Ontario from 2001 to 2007. Best Pract Res Clin Rheumatol. 2012;26:637647.

17. Styron JF, Barsoum WK, Smyth KA, Singer ME. Preoperative predictors of returning to work following primary total knee arthroplasty. J Bone Joint Surg Am. 2011;93:2-10.

18. Walton NP, Jahromi I, Lewis PL, Dobson PJ, Angel KR, Campbell DG. Patient-perceived outcomes and return to sport and work: TKA versus mini-incision unicompartmental knee arthroplasty. J Knee Surg. 2006;19:112-116.

19. Weingarten S, Riedinger MS, Sandhu M, Bowers C, Ellrodt AG, Nunn C, Hobson P, Greengold N. Can practice guidelines safely reduce hospital length of stay? Results from a multicenter interventional study. Am J Med. 1998;105:33-40. 